\title{
The Impact of Socioeconomic Status on Disability Trajectory of the EIderly in China
}

\author{
Meng Wei ${ }^{1, a, *}$ Jun $\mathrm{Li}^{1, \mathrm{~b}}$ and Hongman Wang ${ }^{1, \mathrm{c}}$ \\ ${ }^{1}$ Institute for Medical Humanities, Peking University, Beijing, China \\ a525171868@qq.com, bjjmulj@126.com, cjkshx2017@126.com \\ *corresponding author
}

Keywords: China, Socioeconomic Status, Older Adults, Disability Trajectory, Group-Based Trajectory Mode

\begin{abstract}
Using data from the Chinese Longitudinal Health Longevity Surveys of 2005, 2008 and 2011, the authors analyzed the impact of socioeconomic status (SES) on disability trajectory (DT) by multivariate logistic regression and a group-based model. Analysis shows that three types of DT exist for elders: Type 1, independent; Type 2, low start, rapidly increasing in late life; and Type 3, high start, rapid development. Literate elderly and elderly with higher occupational status are more likely to be assigned to a higher level DT type. Poor economic status (ES) always aggravates the development trend of DT. When designing programs preventing disability of older adults, differences of DTs should be considered.
\end{abstract}

\section{Introduction}

Currently, the populations of most countries are aging rapidly, not only of developed countries but also of developing ones. China has more elderly than any other country. By the end of 2015, the number of people aged 65 years and older was 144 million, 10.5\% of the population [1]. Family size decreases due to a low birthrate, in spite of various birth stimulus policies. Better healthcare driven by socio-economic development and the consistent increase of healthcare input extends life expectancy of older Chinese adults who are suffering from chronic diseases. However, this also leads to an increasing disability rate among older adults [2] and brings a series of challenges for family care resources, healthcare resources, etc. Caring for the elderly, especially those who are disabled, has become a critical issue.

There is significant heterogeneity of socioeconomic status (SES) within the elderly population in developing countries, such as China. China's transformation to a market-oriented economy, which started in 1978, led to a substantive improvement in its people's living standard. However, this also incurred a marked inequality of SES among different classes and regions, which may cause huge disparities in people's health [3]. The influence of SES on late-life health is a particularly active area of research [4]. In short, SES has been shown to be a potent predictor of health status of the elderly, and a higher SES can improve an individual's health status directly or indirectly [5].

SES consists of three dimensions: educational attainment, occupational status and economic income [6]. Educational attainment reflects the ability of a person to obtain social, psychological and economic resources; occupational status reflects a person's social status, rights, responsibilities, physical activity and health risks; economic income reflects a person's consumption capacity, housing conditions, and access to nutrition and healthcare resources. The connection between socioeconomic indicators and disability is clearly of importance within the aging literature.

Higher economic status (ES) is uniformed proved to be significantly associated with lower risk of functional impairment [7].

The relationship between educational attainment and the activity of daily living (ADL) of the elderly are controversial, no matter for developed country or developing ones, as shown by previous empirical studies. Higher levels of education were observed in subjects with lower levels of disability in the national surveys in China, India, South Africa, Russia and US [7] and two local studies, one in Wuhan, the capital of a central 
China province, and the other in Taiwan [8]. However, inverse association was also reported, at least in the oldest-old in China [9] (Yi Zeng, 2004), and especially in men [10] (Zimmer et al. 2012).

The association between occupational status and the ADL are not consistent. They are positively correlated in northern Taiwanese [11] and in Italy, but negatively related in China [9], despite the Italian study used loss of instrumental daily functions to measure disability and only farmers and white-collar workers were compared [12].

In addition, previous studies found that biological and social factors such as gender, ethnicity, lefthandedness, urban/rural residence, medical treatment and nutritional status at early life significantly affect the ADL of the elderly. Being female [13], of Han ethnicity [14], the ethnic majority in China, left-handed [15], and having poorer medical treatment and nutritional status before old age [9] are associated with greater risk of disability. The negative effect of left-handedness could be possibly explained by genetic reasons or that the current living facilities in China are left-handedness-unfriendly [15]. However, relationship between urban/rural residence and the ADL are controversial [8].

For most people, disability is a dynamic process that evolves over time and is the result of an accumulation of factors across the life course [14]. The trend and process of the change of the level of disability of older adults should not be ignored. In addition, the disability trajectory (DT) of older adults exhibits different patterns not only with a distinct level of disability at the starting and end points but also with constantly changing developments during the process. It is inappropriate to generalize the development of ADL into one trajectory. The group-based trajectory model, which represents a special category of the finite mixture model [16], could effectively fill the gap left by prior DT studies that tend to generalize the development of ADL into a single trajectory and ignore the differences in development.

In most of the prior studies, SES was introduced into models only as a potential confounder, and these studies suggested that there is controversy as to whether or how educational attainment affects the attribution to trajectory type [16]. As to occupational status, prior studies found that elderly who worked in agriculture are more likely to be subordinated to a lower DT type [14].

In summary, prior studies have explored the DT and ADL of older adults from different disciplines and perspectives and are crucially important for contributing to better knowledge and a more accurate evaluation of DT and ADL. However, they also have limitations. Most of the previous studies focused on the influence of ES at old age on the ADL or the influencing factors of DT type of the elderly. Longitudinal empirical study on the effect of ES on development trend of DT is scarce. In order to fill these gaps, the impacts of SES on their DT type and development trend of DT were analyzed.

\section{Study design}

A. Data source

The present study selected data from the last three rounds of the Chinese Longitudinal Health and Longevity Survey, which covered 23 provinces and has been conducted for six rounds in each of 1998, 2000, 2002, 2005, 2008 and 2011. (See the survey website for details: http://web5.pku.edu.cn/ageing/html/detail_project_1.html).

In total, 7,979 older adults in the 2005 survey aged between 65 to 105 years (inclusive) were chosen for the study because the age above 105 might have been wrongly reported.

\section{B. Definition}

A disability trajectory reflects the dynamic trend of the level of disability across the lifetime of older adults. It is frequently examined using the Katz Index of Independence in Activities of Daily Living [17]. An elderly individual would be defined as disabled if he/she required assistance in one of the six following activities of the index: feeding, continence, transferring, going to the toilet, dressing, and bathing. The higher the number of items that required assistance, the higher the level of disability. If a respondent is capable of performing all of the six activities, he/she would be defined as independent.

C. Variables 
According to the theory of group-based trajectory modeling, the time-invariant explanatory variable can predict to which DT type an older adult belongs, time-variant variables can affect the development trend of trajectories.

In order to test the impact of the time-invariant explanatory variables on DT type attribution, the authors selected educational attainment and occupational status as the independent variables and selected gender, ethnicity, left-handedness, urban/rural residence, childhood nutrition, medical treatment at the age of 60 and in childhood, which are fixed attributes of the subjects, as the control variables. The following paragraph explains the reasons in detail.

Education and occupation are determined early in life. Gender and ethnicity are unchangeable demographic characteristics of the individual. Left-handedness become a habitus during childhood or adulthood and thus quite fixed. Urban/rural residence may change over time, but moves in response to disability onset are not common [18].Nutrition and medical status in childhood and medical status at 60 years old, indicating the early life condition of subjects and condition before the old age, respectively, are also quite constant.

D. Methodology

The present study adopts the group-based model of trajectory for the research on DT of older adults, as it could identify clusters of individuals with similar trajectories, distinguish different types of disability development trajectories, identify the features of people of different trajectory types and explain the categorization of trajectory of individuals. The time-variation covariates can also be incorporated into the model to characterize the differences between development trends of trajectories. For theoretical details regarding the model, see Nagin [16]. The following is an introduction of the design of the model used in this study.

Since the independent variable of the present study is count data and there are more zeros than under the Poisson assumption (i.e., zero inflation occurs in terms of the distribution of this count variable at given time points), the authors determined to adopt the group-based zero-inflated Poisson model. To identify the best number of trajectory types, we ran group-based models with different numbers of trajectory types and under different time function combinations, starting from a single type (number of trajectory group $=1$ ), and identified the best number of trajectory types according to the Bayesian information criterion (BIC). We found that when there are 3 trajectory types, the absolute value of the BIC is the lowest. Therefore, the optimum number of trajectory types was identified as 3. Subsequently, we compared the BICs when the time function of the model was designed into different combinations and generated the optimum combination of each group of trajectory type. After running the designed development model, we could obtain an estimate of individual respondents' identity probability of the verified trajectory types, assign individuals to a certain trajectory type (with the highest probability estimate value according to the highest probability allocation principle) and assign the respondents into different DT types on this basis.

After classifying the trajectory types of group members, the authors incorporated time-invariant explanatory variables into the model and used multivariate logistic regression to examine how the timeinvariant variables affect the trajectory type attribution of group members. Furthermore, the authors incorporated the time-variant variables into the model and investigated the influence of time-variant variables on the development trend of trajectories. The basic route is as follows:

\section{Results}

\section{A. DT of the elderly}

As is shown in Figure 1, for all respondents, there are three types of disability trajectories. $34.98 \%$ of older adults belong to Type 1, "the independent type". 54.28\% of older adults belong to Type 2, the "low start, rapidly increasing in late life type". The remaining $10.74 \%$ of older adults belong to Type 3 , the "high start, rapid development type". 


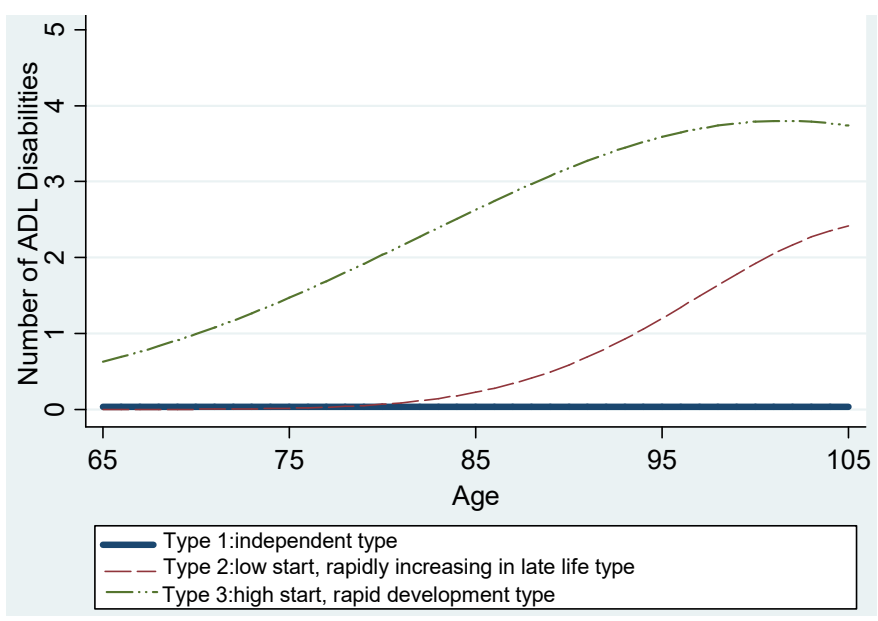

Figure 1. DT of the elderly

B. The impact of SES on DT of the elderly

The impact of SES on the attribution of DT types of the elderly

The authors incorporated time-invariant variables that may influence the DT of the elderly into the model to determine the influencing factors for the attribution of DT types. Among them, educational attainment and occupational status were incorporated as the independent variables and gender, ethnicity, left-handedness, urban/rural residence, childhood nutrition and medical treatment at 60 years old and childhood as the control variables. A multivariate logistic model was used in this study, and the dependent variable was the DT type ( 1 = Type $1,2=$ Type $2,3=$ Type 3$)$. Since the elderly in Type 1 were always relatively independent, and the elderly in Type 2 did not suffer disability until oldest-old age, which were ideal cases, the authors set Type 3 , which has the earliest onset of disability and had the most severe disability, as the reference class.

Table 1. The influencing factors of DT type of the elderly

\begin{tabular}{|c|c|c|c|}
\hline Variable & Variable value & Type 1VS. Type 3 & Type 2 VS. Type 3 \\
\hline Gender & Female & $-0.458 * * *$ & -0.147 \\
\hline Ethnicity & Han & $-0.541 *$ & -0.380 \\
\hline Left- handedness & Yes & $-0.835 * * *$ & $-0.596^{* *}$ \\
\hline Residence & Rural & $0.209+$ & $0.189+$ \\
\hline \multicolumn{4}{|l|}{ Education } \\
\hline & Literate & $-0.295^{*}$ & 0.013 \\
\hline Occupation & Lower occupation & $0.599 * * *$ & 0.187 \\
\hline Childhood nutrition & Inadequate & -0.028 & -0.098 \\
\hline $\begin{array}{l}\text { Timely medical care during } \\
\text { childhood } \\
\text { Timely medical care at } 60 \text { years }\end{array}$ & Yes & 0.063 & -0.087 \\
\hline $\begin{array}{l}\text { old } \\
\text { Constant }\end{array}$ & Yes & $\begin{array}{l}-0.043 \\
1.962 \\
\end{array}$ & $\begin{array}{l}0.169 \\
1.572 \\
\end{array}$ \\
\hline
\end{tabular}


Table 1, cont.

$\triangle \times 2$ $93.39 * * *$

Note: $\triangle x^{2}$ indicates change in chi-square for the total model in comparison with the model with constant only. $* * * \mathrm{p}<0.001, * * \mathrm{p}<0.01, * \mathrm{p}<0.05,+\mathrm{p}<0.1$

The results of the regression analysis show that educational attainment, occupational status, gender, ethnicity, left-handedness and urban/rural residence have significant impact on the distribution of DT types of the elderly. However, childhood nutrition and medical treatment at 60 years old and during childhood have no significant impact.

As indicated in Table 1, illiterate older adults are more likely to be assigned to Type 1 than literate older adults, and educational attainment has no significant impact on the distribution of Type 2 or Type 3 . Older adults with low occupational status are more likely to be assigned to Type 1 than those with high occupational status, and occupational status has no significant impact on the distribution of Type 2 or Type 3 .

In addition, after controlling other variables, older women are less likely to fall into Type 1 than older men. Gender has no significant impact on the distribution of Type 2 or Type 3. The elderly of Han ethnicity are less likely to fall into Type 1 than are minorities. Ethnicity has no significant impact on Type 2 or Type 3 . Left-handed older adults are less likely to fall into Type 1 and Type 2 than are right-handed older adults. The probability of rural older adults being assigned to Type 1 and Type 2 is higher than that of urban older adults.

Predictive effect of SES at old age on the development trend of DT

According to the theory of group-based trajectory modeling, time-variant variables can affect the development trend of the trajectory. That is, under different conditions, the development speed of the same trajectory may accelerate or slow down. Older adults with better ES often have more healthcare resources, which are conducive to maintaining their ADL. However, whether or not the ES at old age could impact the development trend of DT remains unknown. To answer this question, the authors chose the ES at old age as an important indicator of SES and predicted its impact on the development trend of DT.



Figure 2. The DT with good ES after 65 years old 




Figure 3. The DT with poor ES after 65 years old

The authors drew the DT under two ES conditions and compared the two scenarios: good ES after 65 years old, poor ES after 65 years old. We found the development trends under the two scenarios are distinctively different. ES has little impact on the elderly of Type 1, who have the best physical fitness and the smallest demand for resources to promote health. Unlike the scenario of having good ES after 65 years old, for Type 2 and Type 3, when an older adult has poor ES after the age of 65, the trend goes up with a steeper slope and faster ascending speed, the onset times of medium and severe disability are advanced.

In short, ES has little impact on the independent type, but a great impact on the other two types. Good ES can delay the occurrence and development trend of disability. Conversely, under poor ES, the starting point of disability occurs earlier, the development trend is significantly accelerated and the degree of disability is more severe.

\section{Discussion}

Although there is an increasing body of literature on disability and SES in developed countries, similar research is relatively scarce in developing countries. This study makes new findings concerning the DT and SES of old age in developing countries. Research on the health of the elderly in China, which is the largest developing country and whose aged population is larger than that of any other developing country, is of great reference value to such countries.

The elderly of different DT types suffer disability at different times with different development trends. To rationally arrange and maximize the use of care resources and delay the occurrence and mitigate the development speed of disability, different response measures at different points should be taken for different DT types. With reference to public health actions for different periods in life as referenced in the "World Report on Aging and Health 2015' issued by the World Health Organization, the authors propose public health actions that might be taken at different points for the three trajectory types. It should be noted that the trajectory types do not necessarily represent the development process of disability of each individual older person, and the action points are not strictly prescribed. Instead, the proposals refer to the general timing for points of public health action.

The independent type can maintain their ADL despite advancement of age. Therefore, older persons of Type 1 should devote time to maintaining or strengthening their ADL and preventing or controlling the occurrence and development of chronic diseases (see Figure 4). 


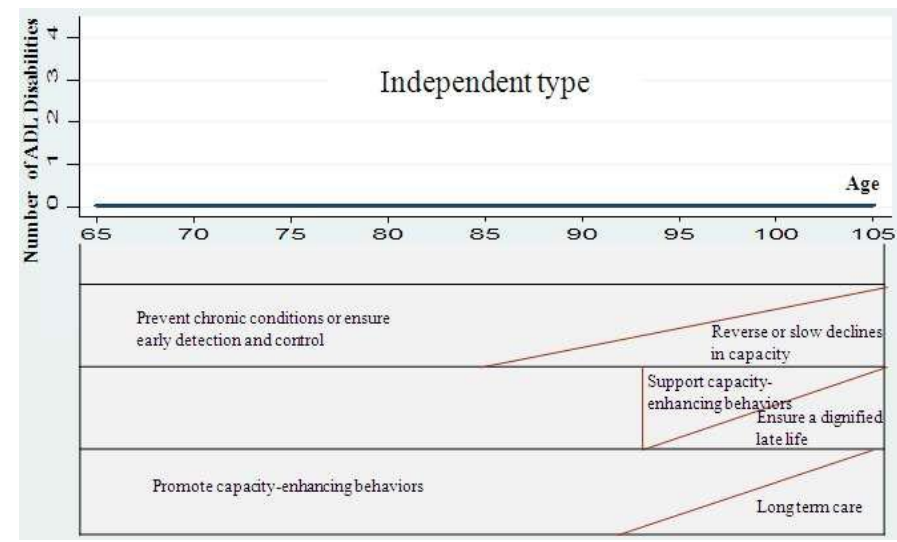

Figure 4. Public health action for Type 1 older adults

Type 2 older adults remain in a status of mild disability (i.e., cannot shower or use the toilet independently) during the age of 80-100 and begin to experience medium disability after age 100. Although the decline in ADL of Type 2 is not serious, public health action should be taken earlier compared to that for the independent type (see Figure 5). Prior studies have demonstrated that a livable environment and support facilities could play a significant role in the adaptation of elderly individuals to mild disability [19]. For older adults who remain mildly disabled during most of their late life, if we could raise social awareness regarding the linkage between a livable community and elderly health and reduce the barriers that elderly individuals face in daily life through appropriate technology, we might expect a substantial reduction of the disability level of the elderly.

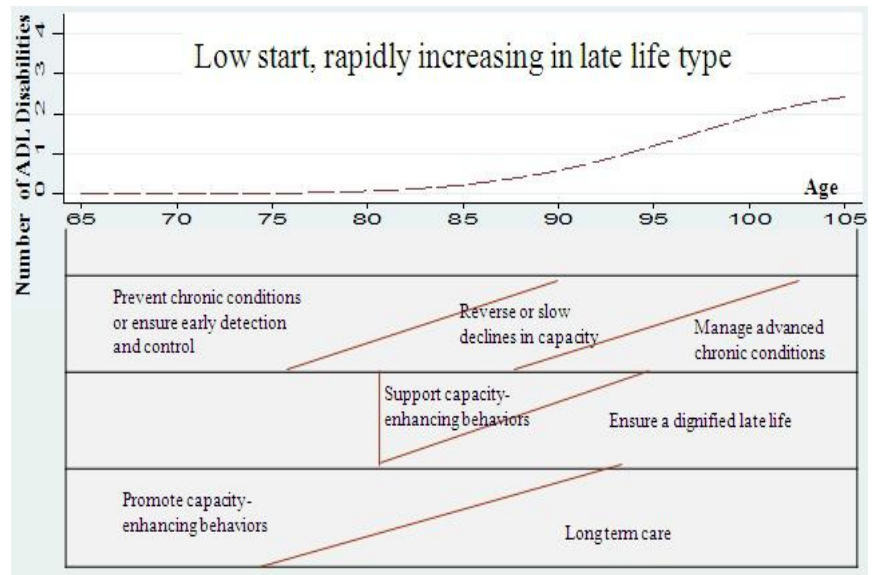

Figure 5. Public health action for Type 2 older adults

Type 3 older adults, who suffer mild disability at the age of 65 and shift to medium or severe disability after the age of 80, are disadvantaged in terms of income, healthcare and other resources. More social resources, such as care assistance, economic support and medical-care services, should be provided. For the timing of public health points of action, all aspects of planning should be advanced earlier than is recommended for Type 2. The time spent on long-term care and management of serious chronic diseases would be considerably greater for Type 3 than the other two types. Specific action items and time point planning are shown in Figure 6. 


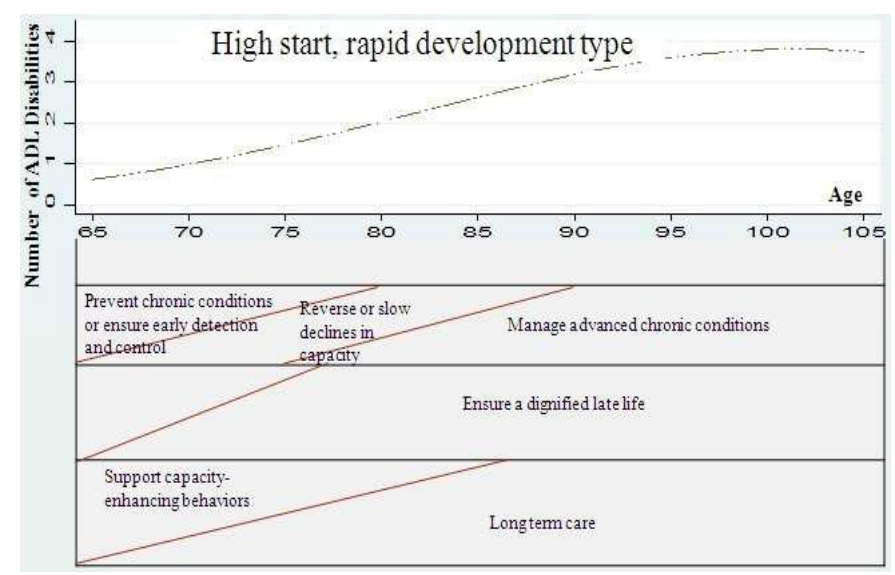

Figure 6. Public health action for Type 3 older adults

Educational attainment and occupational status significantly affect the type distribution of DT of the elderly. Disability risk increases in the elderly who have had some schooling and worked in occupations of higher status. This result conforms to the finding of Zimmer et al.'s [10]. However, it differs from the findings of Biritwum et al. [7], which may be explained by the difference in the scope of data sets or the difference of definition of disability.

On one hand, illiterate older adults and older adults working in occupations of lower status were mostly manual workers and thus have a better ADL. Older adults who have had some schooling and work in occupations of higher status mainly worked indoors, and the resulting lack of exercise makes them susceptible to various chronic vascular diseases, which is detrimental to maintaining their ADL. Compared with developed countries that took more time to reach their current level of development, the economies of developing countries such as China have developed at a faster rate without a commensurate increase in health consciousness among its people. At present, the frequent occurrence of health problems among intellectuals in developing countries has proven this point. More seriously, this phenomenon is plaguing more and more younger people [20]. Developing countries should not ignore the cultivation of health consciousness among their people when accelerating their socio-economic development.

On the other hand, the elderly with certain educational attainment and occupations of higher status can benefit from good ES and healthcare and can often survive with disabilities. However, illiterate older adults and older adults with occupations of lower status, because of low healthcare awareness and poor access to healthcare, often don't live long with disabilities. In the future, as noted by previous studies [21], for the improvement of the health of the older population in China and elsewhere, growth in education should be considered. Furthermore, we also suggest that health equity be used as a measure of social development and progress.

The results of this study demonstrate the effect of ES on the development trend of DT of the elderly. Although the type of DT at old age might has been fixed by conditions at early stage, ES at old age can still influence the development trend of DT, and poor ES can aggravate disabilities. Social Causality Theory argues that the health of individuals is limited by social structural factors: the lower the SES, the worse the health status [22]. This finding further validates the Social Causal Theory. However, compared with younger individuals, older individuals often have lower ES, which hinders their access to quality healthcare resources. This statement is particularly accurate for certain developing countries that are less economically developed and unable to provide adequate social security.

This study has several strengths. First, the authors propose public health actions that might be taken at different points for the three trajectory types. Compared with the "World Report on Aging and Health 2015", our proposal is more specific in terms of the actions and their implementing time points for older people of different DTs. Second, most of the previous studies focused on the influence of ES at old age on the ADL or 
the influencing factors of DT type of the elderly but ignored the effect of ES on the development trend of DT. This paper contributes to filling this gap.

Our research also has limitations. First, because of a limited sample size for the oldest of the elderly, the confidence interval regarding this group's disability trajectory is occasionally wide. Second, in theory, genetic factors have a significant impact on the DT of the elderly. However, because our access to the genetic data of the respondents was limited, we did not consider the impact of genetic factors.

\section{References}

[1] Ministry of Civil Affairs of the People's Republic of China. Social service development statistics bulletin for 2015. 2016-7-11. http://www.mca.gov.cn/article/zwgk/mzyw/201607/20160700001136.shtml.

[2] Yunzhong You, Xiaoying Zheng. (2005). Mortality and health of Chinese population. Beijing: Peking University Press.

[3] Xueqiang Wang, Peijie Chen. (2014). Population ageing challenges health care in China. The Lancet, 3 : 870.

[4] Lynch, S. M. (2008). Race, socioeconomic status and health across the life course: Introduction to the special issue. Research on Aging, 30: 127-136.

[5] Adler N.E., Ostrove J.M. (1999). Socioeconomic status and health: what we know and what we don't. Annals of the New York Academy of Sciences, 896: 3-15.

[6] Duncan O.D. (1961). A socioeconomic index for all occupations. In Reiss AJ(Ed.). Occupations and Social Status. New York: Free Press.

[7] Biritwum R.B., Minicuci N., Yawson A.E., et al. (2016). Prevalence of and factors associated with frailty and disability in older adults from China, Ghana, India, Mexico, Russia and South Africa. Maturitas, 91: 8-18. [8] Jersey Liang, Xian Liu, Shengzu Gu. (2001). Transitions in functional status among older people in Wuhan, China: socioeconomic differentials. Journal of Clinical Epidemiology, 54: 1126-1138.

[9] Yi Zeng, Yuzhi Liu, Zhenyu Xiao, Chunyuan Zhang (2004). The socio-demographic and healthy status of the oldest-old in China. Chinese Journal of Population Science, SI: 4-13.

[10] Zachary Zimmer, Linda G. Martin, Daniel S. Nagin, Bobby L. Jones. (2012). Modeling disability trajectories and mortality of the oldest-old in China. Demography, 49: 291-314.

[11] Li, C Y, Wu, S C, Wen, S W. (2000). Longest held occupation in a lifetime and risk of disability in activities of daily living. Occupational and Environmental Medicine, 57: 550-554.

[12] Cristina Geroldi, Giovanni B. Frisoni Renzo Rozzini, Marco Trabucchi. (1996). Disability and principal lifetime occupation in the elderly. The International Journal of Aging and Human Development, 43: 317-324.

[13] Kabir, Z. N., Carol Tishelman, Hedda Agüero-Torres, A.M.R.Chowdhury, Bengt Winblad, Bengt Höjer (2003). Gender and rural-urban differences in reported health status by older people in Bangladesh. Archives of Gerontology and Geriatrics, 37: 77-91.

[14] Xiwei Wu. (2009). The types of disability trajectory of the oldest-old in China: an application of group based development modeling. Population Research, 4: 54-65.

[15] Danan Gu, Yuzhi Liu, Yingxin Zhang, Jie Zhou. (2008). Biomarker and healthy longevity among the Chinese elderly: the case of left-handedness. Chinese Journal of Population Science, 4: 37-43.

[16] Nagin, D. S. (2005). Group-based modeling of development. Harvard University Press. Cambridge, MA. [17] Katz, S., et al. (1963). Studies of illness in the aged: the index of ADL: a standardized measure of biological and psychosocial function. Journal of the American Medical Association, 12.

[18] Zhu, J. (1998). Rural out-migration in China: a multilevel model. In R. E. Bilsborrow (Ed.), migration, urbanization, and development: new directions and issues. Norwell, MA: Kluwer Academic Publishers.

[19] May A. Beydoun, Barry M. Popkin. (2005). The impact of socio-economic factors on functional status decline among community-dwelling older adults in China. Social Science \& Medicine, 60: 2045-2057. [20] Zhanjia Zhang, Bing Zhang. (2015). The physical health condition and changing tendency of college staff- take Tsinghua University as an example. Sports Research and Education, 1: 41-44. 
[21] Yaohui Zhao, James P Smith, John Strauss. (2014). Can China age healthily? The Lancet, 384: 723-724. [22] Dahl, Espen. (1996). Social mobility and health: cause or effect? British Medical Journal, 313: 435-436. 